\title{
ESTIMAÇÃO BAYESIANA DE PARÂMETROS GENÉTICOS DE PESOS CORPORAIS EM UM REBANHO DA RAÇA GUZERÁ
}

\author{
COSTA, Maria Teresa Galdiano Pimenta ${ }^{1}$ \\ SANCHES, Adhemar ${ }^{2}$ \\ MUNARI, Danísio Prado ${ }^{2}$
}

Recebido em: 2009-03-23

Aprovado em: 2009-04-29

Issue DOI: $10.3738 / 1982.2278 .182$

\begin{abstract}
RESUMO: Foram analisados dados de 1796 animais, nascidos no período de 1978 a 1996, do rebanho Guzerá, do Campus da UNESP, Ilha Solteira, SP para se obter estimativas de componentes de variância, coeficientes de herdabilidade e correlações genéticas, considerando os pesos aos 12, 18 e 24 meses de idade. Os componentes de variância, as herdabilidades e os valores genéticos dos animais foram estimados pelos métodos de Máxima Verossimilhança Restrita (REML) e Bayesiano, com o objetivo de avaliar propriedades das estimativas obtidas por ambas metodologias. As correlações genéticas foram obtidas pelo procedimento CORR do SAS, utilizando-se os valores genéticos dos animais obtidos pelos dois métodos. O modelo estatístico incluiu o efeito fixo de grupo de contemporâneos e os efeitos aleatórios genético aditivo direto, genético materno, de ambiente permanente da vaca e residual. As estimativas das herdabilidades diretas obtidas pelo método REML foram de 0,09, 0,08 e 0,12 e pelo Bayesiano foram de 0,07, 0,08 e 0,11, as herdabilidades maternas encontradas pelo REML foram de 0,08 , 0,04 e 0,07 e pelo Bayesiano de 0,07, 0,04 e 0,06, respectivamente, para os pesos aos 12, 18 e 24 meses de idade. Os valores das correlações genéticas foram altos e semelhantes nos dois métodos estudados. Os comprimentos dos intervalos de confiança obtidos pelo REML foram maiores do que os dos intervalos de credibilidade obtidos pelo Bayesiano, sugerindo que o método Bayesiano fornece resultados mais precisos que o método REML.
\end{abstract}

Palavras-chave:Variância genética. Herdabilidade. Correlação genética. Método Bayesiano. Método REML.

SUMMARY: Data from 1796 animals born between 1978 and 1996 in the Guzera herd of UNESP, Ilha Solteira, SP, were analysed to obtain variance components, heritabilities and genetic correlation for weights at 12,18 and 24 months of age. The variance components, the heritabilities and the animals breeding values were estimated by the Restricted Maximum Likelihood (REML) and Bayesian methods, with the aim of evaluate properties of the estimates from both methods. The genetic correlations were obtained by CORR procedure of SAS, using the animal breeding values destimated from both methods. The statistic model for the weights at 12, 18 and 24 months, included fixed effects of contemporary groups and the random effects direct additive genetic, maternal genetic, permanent environmental of the cow and residual. The estimates of direct heritabilities were $0.09,0.08$ and 0.12 from REML and 0.07, 0.08 and 0.11 from Bayesian method, the estimates of maternal heritabilities were 0.08, 0.04 and 0.07 from REML and 0.07, 0.04 and 0.06 from Bayesian method, respectively, for weights at 12, 18 and 24 months of age. In both methods the estimates of genetic correlations coefficients were high and similar. The lengths of the confidence intervals from REML were larger than the credibility intervals from Bayesian, suggesting that the Bayesian method gives results with better precision than REML method.

Key words: Variance components. Heritabilities. Genetic correlation. Bayesian method. REML method.

\footnotetext{
1 Engenheira Agrônoma - MSc em Genética e Melhoramento animal. Prof. FAFRAM. E-mail: mteresagp@,feituverava. $\underline{\text { com.br }}$

2 Professores do Departamento de Ciências Exatas - UNESP - Campus Jaboticabal. E-mail: adhesan@,fcav.unesp.br; dani$\underline{\text { sio@,fcav.unesp.br. }}$
}

Nucleus Animalium, v.1, n.1, maio 2009 


\section{INTRODUÇÃO}

Guzerá é uma raça considerada de duplo propósito, apta para a produção de leite e de carne, tem ocupado um papel de destaque entre os zebuínos e vem recebendo atenção de grupos de pesquisas.

Sendo a menos exigente das raças zebuínas, a raça Guzerá é considerada como material genético mais apropriado para regiões brasileiras, onde as adversidades ambientais e de manejo são maiores. Tem sido selecionada desde 1977 no Campus da UNESP de Ilha Solteira, SP, que encampou o desafio de promover o seu melhoramento genético.

A obtenção de progresso genético depende do uso adequado da variação genética das características sob consideração. Assim, é necessário o conhecimento prévio dos parâmetros genéticos das características de interesse, para que a variação genética seja eficientemente utilizada em um programa de seleção, pois destes irão depender a predição das respostas direta e correlacionada à seleção e a definição do método de seleção mais apropriado. Daí decorre a relevância de serem estudados métodos de estimação de maior eficiência, sendo estes temas de permanente interesse no melhoramento animal. Com a facilidade de acesso a computadores de grande capacidade de trabalho, aliada a utilização de potentes softwares com algoritmos especializados para estimação de parâmetros, tem ocorrido grande progresso nas pesquisas em ciência animal, particularmente, no melhoramento genético.

Procedimentos, que forneçam estimativas cada vez mais acuradas e que apresentem propriedades de interesse na estimação de componentes de variância, estão sendo muito requisitados, e uma opção interessante é o método Bayesiano.

A metodologia Bayesiana diferencia-se da metodologia estatística clássica ou frequentista, pois os parâmetros são vistos como variáveis aleatórias cujo comportamento é regulado por uma distribuição de probabilidade que se assume sobre seus possíveis valores, traduzindo uma informação inicial (ou a priori) que se tenha sobre os parâmetros, antes mesmo de se obter os dados. A seguir, essa informação a priori é combinada com aquela proveniente dos dados, produzindo uma informação a posteriori sobre os parâmetros. Essa informação $a$ posteriori se expressa na chamada distribuição a posteriori dos parâmetros, que é utilizada para se fazer inferência sobre os mesmos.

Neste trabalho utilizando dados do rebanho Guzerá do Campus da UNESP, Ilha Solteira, SP, foram obtidas estimativas de componentes de variância, coeficientes de herdabilidade e correlações genéticas, considerando os pesos aos 12, 18 e 24 meses de idade, pelos métodos de máxima verossimilhança restrita e Bayesiano, com o objetivo de avaliar as propriedades das estimativas obtidas por ambos os métodos. 


\section{REVISÃO DE LITERATURA}

Componentes de variância são parâmetros correspondentes às variâncias dos efeitos aleatórios de um modelo estatístico e, em ciências agrárias, são largamente utilizados no melhoramento genético animal e vegetal. Entre os métodos de estimação de componentes de variância citados na literatura, o desenvolvido por Fisher, de análise de variância, foi o que impulsionou os outros métodos de estimação (SEARLE et al., 1992).

O método REML, segundo Lopes et al. (1993) e Eler(1994), considera a função densidade de probabilidade das observações em duas partes independentes, uma referente aos efeitos fixos e outra aos aleatórios. As estimativas dos componentes de variância são obtidas a partir da derivação do logaritmo da parte referente aos efeitos aleatórios em relação aos componentes de variância, eliminando-se, desta forma, o viés decorrente do uso do método ML, resultante da não consideração da perda de graus de liberdade na estimação dos efeitos fixos do modelo.

Entre os programas computacionais, capazes de efetuar análises para estimação de componentes de (co)variância, utilizando os processos DFREML e REML-EM, o software MTDFREML (Multiple Trait Derivative Free Restricted Maximum Likelihood) estima os referidos componentes de (co)variância, usando modelo animal, em análises uni e multicaráter. Além disso, utiliza o método Simplex como estratégia de procura do máximo da função de verossimilhança e as rotinas SPARSPAK e decomposição de Choleski para matrizes esparsas (BOLDMAN et al., 1993).

Nametodologia Bayesiana, especialmente, quando os processos analíticos são complexos, utiliza-se um processo de amostragem da distribuição a posteriori, chamado de amostragem de Gibbs, que faz parte de um conjunto de processos iterativos, denominados de métodos de Monte Carlo baseados em Cadeias de Markov, ou MCMC (Markov Chain Monte Carlo). Segundo Van Tassel et al. (1995) o uso da amostragem de Gibbs permite análise de conjunto de dados maiores que os permitidos na metodologia REML. Mesmo utilizando rotinas para resolução de matrizes esparsas, propicia estimativas diretas e acuradas dos componentes de (co)variância e valores genéticos, bem como dos intervalos de confiança (no contexto Bayesiano, denominados de intervalos de credibilidade) da estimativa e pode ser usado em computadores de médio porte, devido à pequena quantidade de informação que mantém na memória. Tais características dão certa vantagem ao método de Amostragem de Gibbs, no contexto Bayesiano, em relação ao método REML.

Esses métodos, assim como outros, têm sido comparados entre si em diversas aplicações. Por exemplo, Wright et al. (2000) realizaram comparações entre análises de experimentos de seleção, em melhoramento animal, conduzidas pelos métodos REML e Bayesiano. Esses autores mostraram que os resultados da seleção, cujos componentes de variância foram provenientes do método Bayesiano, diferenciou-se da análise tradicional efetuada pelo REML, levando, consequentemente, a outras decisões importantes na seleção de indivíduos para melhoria das próximas gerações.

Nucleus Animalium, v.1, n.1, maio 2009 
Oliveira et al. (2000) estimaram componentes de variância, em características de crescimento na raça Canchim, utilizando os métodos REML e Bayesiano, e concluíram que ambos os métodos forneceram estimativas de componentes de variância semelhantes. O mesmo foi observado por Oliveira et al. (2002), em animais da raça Nelore, que comparando seus resultados obtidos pelos dois métodos, concluíram que o método Bayesiano proporcionou estimativas semelhantes às disponibilizadas pela metodologia de máxima verossimilhança restrita.

Segundo Falconer e Mackay (1996), a herdabilidade expressa a proporção da variância total que é atribuída aos efeitos médios dos genes, e são estes que determinam o grau de semelhança entre parentes. Segundo Van Vleck et al. (1987), é específica da população e da característica considerada e, se a variância genética para a mesma característica em duas populações difere, a herdabilidade também irá diferir. Além do método de estimação, vários fatores podem afetar as estimativas de herdabilidade, como: raça, sexo, manejo alimentar e origem dos dados.

Segundo Koots et al. (1994), tanto para a pesquisa como para a prática em melhoramento genético animal, estimativas de componentes de (co)variâncias e herdabilidades são itens essenciais. Estes parâmetros caracterizam uma população e podem mudar com o tempo, devido a mudanças na estrutura genética da população e decisões de manejo.

Em bovinos Guzerá, herdabilidades diretas variando entre 0,08 e 0,13 para peso aos 12 meses foram obtidas por Oliveira (1994); Oliveira et al. (1995) e Mucari (2002). Valores superiores de, respectivamente, 0,32 e 0,24 foram encontrados por Barbosa et al. (1999) e Souza et al. (2002). Herdabilidades maternas de 0,07 e 0,12, para o referido peso, foram estimadas por Mucari (2002) e Souza et al. (2002), respectivamente.

Para pesos aos 18 meses de idade, em bovinos da raça Guzerá, Martins Filho et al. (1997) e Mucari (2002) relataram estimativas de herdabilidade direta iguais a 0,12 e 0,08, respectivamente. Barbosa et al. (1999) e Souza et al. (2002) encontraram valores de herdabilidade mais altos, iguais a 0,56 e 0,40 , respectivamente. Herdabilidades maternas para esse peso de 0,04 foi relatada por Mucari (2002).

Oliveira et al. (2000) observaram que o método REML forneceu estimativas semelhantes às estimativas (médias a posteriori) obtidas pelo método Bayesiano, para componentes de variância e herdabilidade de pesos em um rebanho da raça Canchim. Os valores de herdabilidade direta, em modelos unicaracter para peso aos 12 meses, foi de 0,40, tanto pelo REML como pelo método Bayesiano, e de 0,34 e 0,38, para peso aos 18 meses, respectivamente, pelos dois métodos.

Para pesos aos 24 meses de idade, em rebanhos Guzerá, Oliveira et al. (1994) obtiveram herdabilidade direta de 0,30 (para fêmeas) e Mucari (2002) relatou valores para herdabilidade direta e materna de 0,12 e 0,07 , respectivamente.

A correlação genética entre duas características indica a extensão em que os mesmos genes afetam a expressão das mesmas, é a correlação entre os valores genéticos de duas 
características. O sentido e a magnitude existentes na relação entre as características são de grande importância no melhoramento genético, pois a mudança decorrente do processo de seleção no valor genético de uma característica pode causar alterações nos valores das demais que são correlacionadas. $\mathrm{O}$ valor genético representa a somatória das contribuições genéticas aditivas de cada loco (VAN VLECK et al., 1987).

Para o rebanho Guzerá da UNESP de Ilha Solteira, Oliveira et al. (1994) estimaram em 0,79 a correlação genética entre os pesos aos 18 e 24 meses de idade de fêmeas e Mucari (2002), observou valores de correlações genéticas iguais a $0,69,0,80$ e 0,81 entre os pesos aos 12 e 18 , 12 e 24 e 18 e 24 meses de idade, respectivamente.

Malhado et al. (2002) obtiveram estimativas de correlação genética de 0,65 entre os pesos aos 12 e 18 meses de idade, para um rebanho da raça Guzerá.

\section{MATERIAL E MÉTODOS}

Os dados utilizados no presente trabalho foram provenientes de animais da raça Guzerá criados na Fazenda de Ensino e Pesquisa da UNESP, Campus de Ilha Solteira-SP, localizada no município de Selvíria, Mato Grosso do Sul. A classificação climática AW permite enquadrar a região onde se localiza, como tropical de inverno seco, que é característico das zonas de cerrado.

O rebanho foi criado em regime de pasto e, por ocasião da seca, recebeu uma suplementação de volumoso (silagem de milho), além de sal mineralizado durante todo o ano. As fêmeas lactantes permaneceram nas pastagens a maior parte do tempo, sendo recolhidas ao estábulo para arraçoamento. Diversas forrageiras foram utilizadas nas pastagens predominando, no entanto, espécies de Braquiária (Brachiaria sp).

Nesse rebanho, o manejo reprodutivo incluiu a inseminação artificial e a monta natural controlada.

Os bezerros eram pesados ao nascer e, posteriormente, a cada três meses, até que os machos completassem 36 meses de idade e as fêmeas atingissem o peso requerido para a reprodução (320 Kg de peso vivo).

As características estudadas foram os pesos aos 12 (P12), 18 (P18) e 24 (P24) meses de idade. Para as análises estatísticas, foi utilizado o arquivo organizado por Mucari (2002).

As análises para P12, P18 e P24 foram realizadas com base em 1796 observações, contendo a identificação do animal, a filiação (identificação de pai e mãe), o grupo de contemporâneos do animal e as classes da idade da vaca ao parto.

As análises para a estimação dos componentes de variâncias e dos coeficientes de herdabilidades foram efetuadas para cada característica separadamente (univariada), utilizando o software MTDFREML, desenvolvido por Boldman et al. (1993) e o software MTGSAM, desenvolvido por Van Tassel e Van Vleck (1995).

Nucleus Animalium, v.1, n.1, maio 2009 
O modelo para os pesos aos 12, 18 e 24 meses, incluiu o efeito fixo de grupo de contemporâneos e os efeitos aleatórios genético aditivo direto, genético materno, de ambiente permanente da vaca e residual.

O procedimento adotado para o cálculo do REML foi utilizar os valores das estimativas dos componentes de variância obtidos por Mucari (2002), como valores iniciais nas análises univariadas.

Foram, também, calculados intervalos de confiança para as estimativas de herdabilidades e correlações entre os efeitos genéticos aditivos diretos e maternos obtidos pelo método REML. Além de propiciar uma melhor descrição das estimativas dos parâmetros, os intervalos de confiança têm como objetivo fornecer informação sobre a precisão das estimativas obtidas. Foi utilizada a seguinte fórmula para o cálculo dos intervalos de confiança (IC) para um parâmetro em particular, simbolizado por $\theta$ :

$\mathrm{IC}=\hat{\theta} \pm \mathrm{k} . \mathrm{EP}(\hat{\theta})$

$\hat{\theta}=$ estimativa do parâmetro $\theta$;

$\mathrm{k}$ = valor obtido na tabela da "distribuição normal padrão" para a confiança especificada;

$\mathrm{EP}=$ erro padrão para a estimativa do parâmetro $\theta$.

O MTGSAM é um conjunto de programas, em FORTRAN, para se fazer estimação Bayesiana de parâmetros de interesse. Utiliza o processo de amostragem de Gibbs ("Gibbs Sampling”), que é um método iterativo de geração de dados de distribuições multidimensionais, com os quais é possível se fazer inferências sobre densidades marginais ou conjuntas, mesmo quando essas densidades não podem ser avaliadas diretamente.

O método de amostragem de Gibbs (GS) consiste na geração, em sequência, de valores ou vetores de variáveis (os parâmetros, no contexto Bayesiano) da distribuição conjunta dessas variáveis, a partir das densidades (ou distribuições) condicionais (full conditional densities). Essas densidades condicionais são as densidades de cada variável, dadas (ou fixadas) as outras variáveis. No contexto Bayesiano, qualquer uma dessas densidades condicionais é obtida da distribuição a posteriori conjunta dos parâmetros, como a densidade de cada um dos parâmetros, dados (ou fixados) os outros parâmetros e os dados (observações).

No MTGSAM, foram utilizados os valores iniciais das estimativas REML dos componentes de variância obtidos por Mucari (2002), sendo considerada uma única característica (univariada) nas análises. Para cada característica analisada neste trabalho foi utilizado um tamanho de cadeia amostral (cadeia de Gibbs) de 1.000.000 ciclos, com um período de descarte amostral (burn-in) dos 25.000 primeiros ciclos. Desta forma, o tamanho da cadeia amostral obtida pelo programa MTGSAM foi de 975.000 ciclos.

O método de Raftery e Lewis (1992) foi utilizado, em seguida, via programa GIBANAL, sob sistema operacional DOS, desenvolvido por Van Kaam (1997), para verificação da 
convergência das cadeias. O programa GIBANAL indica se existe a necessidade de descartar um número maior de ciclos iniciais (burn-in) e de utilizar um intervalo amostral (skipparameter) específico para obtenção de uma correlação serial de valor baixo entre os ciclos. Assim, o procedimento por meio deste teste determina a convergência da cadeia amostral com base na correlação serial da cadeia.

Intervalos de credibilidade foram determinados para as herdabilidades e as correlações entre os efeitos genéticos aditivos diretos e maternos estimados pelo software MTGSAM, adotando-se probabilidade (o equivalente à confiança da inferência clássica) de 95\%, usando-se o pacote estatístico do SAS (1998). Um intervalo de credibilidade (IC) de (1- $\alpha) \%$ de qualquer das herdabilidades de interesse foi obtido da amostra dos $n$ valores da herdabilidade, obtidos da amostra gerada pelo GS, do seguinte modo: considerou-se todos os $\mathrm{n}$ valores da herdabilidade numa ordem crescente, descartando-se os $(\alpha / 2) \%$ menores e os $(\alpha / 2) \%$ maiores. Assim, o intervalo de credibilidade foi calculado como o intervalo real que vai do mínimo até o máximo dos valores restantes.

Intervalos de credibilidade para outros parâmetros foram construídos de modo similar.

As estimativas das correlações genéticas foram obtidas pelo procedimento CORR (Pearson) do SAS (1998), utilizando-se os valores genéticos das características estudadas (P12, P18 e P24), que foram previamente estimados por meio dos programas computacionais MTDFREML (BOLDMAN et al., 1993) ou MTGSAM (VAN TASSEL e VAN VLECK,1995).

\section{RESULTADOS E DISCUSSÃO}

Na Tabela 1, são apresentadas as estimativas dos componentes de variância dos efeitos genético aditivo direto, genético aditivo materno, de ambiente permanente, residual (ambiente temporário) e fenotípico e do componente de covariância entre o efeito genético aditivo direto e materno para P12, P18 e P24, obtidos pelos métodos REML e Bayesiano, para o rebanho Guzerá de Ilha Solteira - SP. Verificou-se que a maioria das estimativas dos componentes de (co)variância para P12, P18 e P24 obtidas pelo método REML, apresentou valores superiores que as obtidas pelo método Bayesiano, exceto para os valores dos componentes da variância residual.

Nucleus Animalium, v.1, n.1, maio 2009 
TABELA 1 - Estimativas dos componentes de (co)variância para os pesos aos 12 (P12), 18 (P18) e 24 (P24) meses de idade em bovinos Guzerá, Ilha Solteira - SP, obtidos pelos métodos REML e Bayesiano.

\begin{tabular}{llllllll}
\hline \multirow{2}{*}{ Variável } & Método & $\mathrm{s}_{a}^{2}$ & $\mathrm{~s}^{2}$ & $\mathrm{~s}_{\text {mat }}^{2}$ & $\mathrm{~s}^{2}$ & $\mathrm{~s}_{e}^{2}$ & $\mathrm{~s}^{2}$ \\
\hline \multirow{2}{*}{ P12 } & REML & 76,21 & 66,46 & 1,72 & 44,77 & 663,75 & 852,91 \\
& BAYESIANO & 54,30 & 56,85 & 13,72 & 36,67 & 685,47 & 847,01 \\
\hline \multirow{2}{*}{ P18 } & REML & 99,73 & 51,37 & 50,56 & 21,86 & 1009,81 & 1233,33 \\
& BAYESIANO & 93,76 & 44,16 & 54,37 & 14,79 & 1022,09 & 1229,17 \\
\hline \multirow{2}{*}{ P24 } & REML & 175,57 & 105,51 & 43,81 & 16,91 & 1176,25 & 1518,05 \\
& BAYESIANO & 153,11 & 79,85 & 65,15 & 13,50 & 1199,58 & 1511,19 \\
\hline
\end{tabular}

$\mathrm{S}_{a}^{2}$ - componente de variância de efeito genético aditivo direto, $\mathbf{S}_{m}^{2}$ - componente de variância de efeito genético aditivo materno, $\mathbf{S}_{a m}$ - componente de covariância entre o efeito genético aditivo direto e o materno, $\mathbf{S}_{p}^{2}$ - componente de variância do efeito de ambiente permanente, $\mathbf{S}_{e}^{2}$ - componente de variância do efeito residual, $\mathbf{S}_{f}^{2}$ - componente de variância do efeito fenotípico.

As estimativas dos coeficientes de herdabilidade dos efeitos direto e materno, das correlações entre os efeitos genéticos aditivos diretos e maternos e os intervalos de confiança e de credibilidade a 95\%, respectivamente, obtidos pelos métodos REML e Bayesiano, são apresentados na Tabela 2, para P12, P18 e P24, em bovinos Guzerá, Ilha Solteira - SP.

TABELA 2 - Estimativas dos coeficientes de herdabilidades direta $\left(h_{d}^{2}\right)$ e materna $\left(h_{m}^{2}\right)$, das as correlações entre os efeitos genéticos aditivos diretos e maternos $\left(\mathrm{r}_{\mathrm{am}}\right)$ e dos intervalos de confiança e de credibilidade a 95\% (IC), respectivamente, obtidos pelos métodos REML e Bayesiano para os pesos aos 12 (P12), 18 (P18) e 24 (P24) meses de idade em bovinos Guzerá, Ilha Solteira - SP.

\begin{tabular}{|c|c|c|c|c|c|c|c|}
\hline Variável & Métodos & $h_{d}^{2}$ & IC & $h_{m}^{2}$ & IC & $\mathrm{r}_{\mathrm{am}}$ & IC \\
\hline \multirow{2}{*}{ P12 } & REML & 0,09 & 0,00 a 0,18 & 0,08 & $-0,01$ a 0,17 & 0,02 & $-0,77$ a 0,81 \\
\hline & BAYESIANO & 0,07 & 0,02 a 0,15 & 0,07 & 0,02 a 0,13 & 0,31 & $-0,40$ a 0,81 \\
\hline \multirow{2}{*}{ P18 } & REML & 0,08 & 0,00 a 0,16 & 0,04 & $-0,02$ a 0,11 & 0,71 & $-0,38$ a 1,80 \\
\hline & BAYESIANO & 0,08 & 0,04 a 0,14 & 0,04 & 0,01 a 0,07 & 0,88 & 0,68 a 0,98 \\
\hline \multirow[b]{2}{*}{ P24 } & REML & 0,12 & 0,02 a 0,22 & 0,07 & $-0,01$ a 0,15 & 0,32 & $-0,47$ a 1,11 \\
\hline & BAYESIANO & 0,11 & 0,05 a 0,19 & 0,06 & 0,02 a 0,11 & 0,63 & 0,07 a 0,92 \\
\hline
\end{tabular}

As estimativas de herdabilidade direta para peso aos 12 meses, obtidas por Oliveira (1994), Oliveira et al. (1995), e Mucari (2002) em rebanhos Guzerá, foram próximos às encontradas no presente trabalho. Para a herdabilidade materna, Mucari (2002) e Souza et al. (2002) encontraram valores semelhantes aos obtidos neste estudo.

A herdabilidade direta estimada para peso aos 18 meses de 0,08 , por ambos os métodos (REML e Bayesiano) foi semelhante, quando comparada com as estimadas por Martins Filho et al. (1997) e Mucari (2002), em rebanhos da raça Guzerá. A herdabilidade materna estimada neste estudo de 0,04 , tanto pelo método REML, quanto pelo Bayesiano, foi de igual valor à 
estimada por Mucari (2002).

Os valores de herdabilidades diretas obtidas por Oliveira et al. (2000), em um rebanho da raça Canchim, para peso aos 12 meses de 0,40 e para peso aos 18 meses de 0,34 e 0,38, respectivamente, obtidos pelos métodos REML e Bayesiano, foram superiores as aqui estimadas por estes dois métodos, para o rebanho Guzerá de Ilha Solteira.

As herdabilidades diretas para peso aos 24 meses de idade de 0,12 e 0,11 , e materna de 0,07 e 0,06 , respectivamente, obtidas pelos métodos REML e Bayesiano, foram semelhantes às obtidas, pelo primeiro método, por Mucari (2002) e inferior a herdabilidade direta obtida por Oliveira et al. (1994), para fêmeas Guzerá .

As baixas estimativas de herdabilidades diretas verificadas neste estudo podem, em parte, ser devida à inclusão dos efeitos maternos no modelo de análise, o que levou a uma diminuição das variâncias genéticas aditivas e, consequentemente, das herdabilidades dos efeitos diretos aditivos.

As correlações genéticas entre os efeitos aditivos direto e materno $\left(\mathrm{r}_{\mathrm{am}}\right)$ para pesos aos 12, 18 e 24 meses de idade, obtidas pelos métodos REML e Bayesiano foram positivas, indicando que as vacas com melhores habilidades maternas teriam filhos com maiores pesos nas idades estudadas.

Os valores negativos encontrados neste estudo dos intervalos de confiança, obtidos pelo método REML, são consequências da aproximação da fórmula utilizada para obter estes intervalos.

$\mathrm{Na}$ Tabela 3, são apresentados os comprimentos dos intervalos de confiança e de credibilidade para as estimativas de herdabilidade direta, herdabilidade materna e correlações entre os efeitos genéticos aditivos diretos e maternos, respectivamente, obtidos para os métodos REML e Bayesiano para os pesos aos 12, 18 e 24 meses de idade em bovinos Guzerá, Ilha Solteira - SP.

TABELA 3 - Comprimentos dos intervalos de confiança e de credibilidade a 95\% (CIC) para as estimativas de herdabilidade direta $\left(h_{d}^{2}\right)$, herdabilidade materna $\left(h_{m}^{2}\right)$ e correlações entre os efeitos genéticos aditivos diretos e maternos $\left(\mathrm{r}_{\mathrm{am}}\right)$, obtidos, respectivamente, para os métodos REML e Bayesiano, para os pesos aos 12 (P12), 18 (P18) e 24 (P24) meses de idade em bovinos Guzerá, Ilha Solteira- SP.

\begin{tabular}{lllll}
\hline \multirow{2}{*}{ Variável } & \multirow{2}{*}{ Métodos } & \multicolumn{2}{l}{ CIC } & $\mathrm{r}_{\mathrm{am}}$ \\
\cline { 3 - 5 } & & $h_{d}^{2}$ & $h_{m}^{2}$ & 1,58 \\
\multirow{2}{*}{ P12 } & REML & 0,18 & 0,18 & 1,21 \\
\hline \multirow{2}{*}{ P18 } & BAYESIANO & 0,13 & 0,11 & 2,18 \\
& REML & 0,16 & 0,13 & 0,30 \\
\hline \multirow{2}{*}{ P24 } & BAYESIANO & 0,10 & 0,06 & 1,58 \\
& REML & 0,20 & 0,16 & 0,85 \\
\hline
\end{tabular}


Os comprimentos dos intervalos de confiança obtidos pelo método REML, no geral, foram maiores do que os intervalos de credibilidade obtidos pelo método Bayesiano. Estes resultados sugerem que o método Bayesiano fornece resultados mais precisos que o método REML.

Na Tabela 4, são apresentadas as estimativas dos coeficientes de correlações genéticas entre os pesos aos 12, 18 e 24 meses de idade, obtidas por meio da correlação linear simples de Pearson entre os valores genéticos estimados pelos métodos REML e Bayesiano.

TABELA 4 - Estimativas dos coeficientes de correlações genéticas entre os pesos aos 12 (P12), 18 (P18) e 24 (P24) meses de idade, obtidas pelos métodos REML (acima da diagonal) e Bayesiano (abaixo da diagonal), em bovinos Guzerá, Ilha Solteira - SP.

\begin{tabular}{llll}
\hline Variável & P12 & P18 & P24 \\
\hline P12 & & 0,68 & 0,79 \\
P18 & 0,74 & & 0,81 \\
& & & \\
P24 & 0,81 & 0,83 & \\
\hline
\end{tabular}

Os valores das estimativas das correlações genéticas foram altos e semelhantes nos dois métodos estudados, indicando que os pesos corporais nas três idades estão altamente associados geneticamente. Assim, grande parte dos genes que influenciam o peso a uma determinada idade também influenciam o peso nas outras idades.

Tanto pelo método REML como pelo método Bayesiano, as estimativas das correlações genéticas entre os pesos estudados, foram semelhantes às estimativas encontradas por Oliveira et al. (1994), entre os pesos aos 18 e 24 meses de idade para fêmeas e por Mucari (2002), entre os pesos aos 12 e 18 meses, 12 e 24 meses e 18 e 24 meses de idade, com ambos autores estudando o mesmo rebanho Guzerá de Ilha Solteira.

Ainda Malhado et al. (2002), em um rebanho distinto da raça Guzerá, encontraram estimativa de correlação genética entre os pesos aos 12 e 18 meses, semelhante com a obtida neste estudo.

\section{CONCLUSÃO}

Considerando que as estimativas dos parâmetros genéticos e classificação dos reprodutores pelos valores genéticos foram semelhantes para ambos os métodos e que o método Bayesiano forneceu resultados mais precisos que o método REML, tendo em vista os comprimentos dos intervalos de confiança e de credibilidade obtidos pelos métodos REML e 
Bayesiano, respectivamente. Recomenda-se a utilização do método Bayesiano para obtenção de estimativas de parâmetros genéticos e dos valores genéticos dos animais.

\section{REFERÊNCIA}

BARBOSA, S. B. P. et al. Fatores ambientais e genéticos que influenciam os pesos corporais de bovinos Guzerá no Estado de Pernambuco. In: REUNIÃO ANUAL DA SOCIEDADE BRASILEIRA DE ZOOTECNIA, 36, 1999, Porto Alegre. Anais... Porto Alegre: SBZ, 1999.

BOLDMAN, K.G. et al. A manual for use of MTDFREML: a set of programs to obtain estimates of variances and covariances (DRAFT). Lincoln: Department of Agriculture/ Agricultural Research Service, 1993. 120 p. 1993.

ELER, J. P. Utilização de modelos animais univariados e multivariados na avaliação genética de bovinos da raça Nelore. 1994. 112p. Tese (Livre-docência) - Universidade de São Paulo. Pirassununga.

FALCONER, D. S.; MACKAY, T. F. C. Introduction to quantitative genetics. 4. ed. Longman, 1996. $463 \mathrm{p}$.

KOOTS, K. R. et al. Analyses of published genetic parameter estimates for beef production traits. 1. Heritability. Animal Breeding Abstract, Edinburg, v.62, n.5, 1994, p.309-338.

LOPES, P. S. et al. Estimação de componentes de variância. Viçosa: UFV, 1993, 61p.

MALHADO, C.H.M.et al. Correlações genéticas, fenotípicas e de ambiente entre os pesos de várias idades em bovinos da raça Guzerá no Estado de São Paulo. Arquives of Veterinary Science, v. 7, n.1, p. 71-75, 2002.

MARTINS FILHO, R. et al. Parâmetros genéticos e fenotípicos de pesos e ganhos em pesos de bovinos zebus no Estado do Ceará. In: REUNIÃO ANUAL DA SOCIEDADE BRASILEIRA DE ZOOTECNIA, 34, 1997, Juiz de Fora. Anais... Juiz de Fora: SBZ, 1997. p. 248-250.

MUCARI, T. B. Estimativas de parâmetros genéticos de pesos em um rebanho Guzerá. 2002.76 p. Dissertação (Mestrado). Faculdade de Ciências Agrárias e Veterinárias. Universidade Estadual Paulista. Jaboticabal.

OLIVEIRA, C. A. L. et al. Estimação de componentes de variância, em características de

Nucleus Animalium, v.1, n.1, maio 2009 
crescimento, utilizando-se Máxima Verossimilhança Restrita e Inferência Bayesiana. In: SIMPÓSIO NACIONAL DA SOCIEDADE BRASILEIRA DE MELHORAMENTO ANIMAL, 3. 2000, Belo Horizonte. Anais... Belo Horizonte: SBMA, 2000.

OLIVEIRA, C. A. L. et al. Avaliação genética para características de crescimento em animais da raça nelore utilizando Inferência Bayesiana. In: SIMPÓSIO NACIONAL DA SOCIEDADE BRASILEIRA DE MELHORAMENTO ANIMAL, 4. 2002, Campo Grande. Anais... Campo Grande: SBMA, 2002.

OLIVEIRA, J. A. Mudanças genéticas e de ambiente em características de crescimento de bovinos Guzerá. Cultura Agronômica, Ilha Solteira, v. 3, n. 1, p 1-8, 1994.

OLIVEIRA, J. A., BEZERRA, L. A. F., BASTOS, J. F. P. Correlações genéticas e fenotípicas entre pesos e produção de leite na primeira lactação em fêmeas da raça Guzerá. Boletim Indústria Animal, Nova Odessa, v. 51, n. 1, p. 7-11, 1994.

OLIVEIRA, J. A.; LÔBO, R. B.; OLIVEIRA, H. N. Tendência genética em pesos e ganhos em peso de bovinos da raça Guzerá. Pesquisa Agropecuária Brasileira, Brasília, v. 30, n. 11, p. 1335-1360, 1995.

RAFTERY, A. E.; LEWIS, S.M. How many iterations in the Gibbs sampler? In: Bayesian Statistics, Oxford University Press, Oxford, U.K., v. 4. 763p.1992.

SAS institute Inc., SAS/STAT. User's Guide, version 6.12, 4. ed, v.2, Cary: SAS Institute Inc., 1998. 842p.

SEARLE, S. R.; CASELlA, G.; MCCULLOCH, C. E. Maximum Likelihood (ML) and Restricted Maximum Likelihood (REML). In: VARIANCE Components. Ithaca: John Wiley \& Sons. Inc, 1992. p. 232 - 257.

SOUZA, J. C. et al. Estimativas de parâmetros genéticos dos pesos aos 205, 365 e 550 dias de idade em bovinos da raça Guzerá na micro-região de Araraquara. In: IV SIMPÓSIO NACIONAL DA SOCIEDADE BRASILEIRA DE MELHORAMENTO ANIMAL, 2002, Campo Grande. Anais... Campo Grande: SBMA, 2002.

VAN KAAM, J. B. C. H. M. GIBANAL: analyzing program for Markov Chain Monte Carlo sequences. Version 2.3, 1997.

VAN TASSELL, C. P.; VAN VLECK, L. D. A manual for use of MTGSAM. A set of 
FORTRAN programs to apply Gibbs sampling to animal models for variance component estimation [DRAFT]. U.S. Departament of Agriculture, Agricultural Research Service. 1995.

VAN TASSEL, C. P.; CASELLA, G.; POLLAK, E. J. Effects of selection on estimates of variance componentes using gibbs sampling and restricted maximum likelihood. Journal Dairy Science, v. 78, p. 678-692, 1995.

VAN VLECK, L. D.; POLLAK, E. J.; OLTENACU, E. A. B. Genetics for the animal sciences. W.H. Freeman and Company, New York, 1987,383p.

WRIGHT, D. R.; H. S. STERN; P. J. BERGER. Comparing traditional and Bayesian analysis of selection experiments in animal breeding. Journal of Agricultural, Biological and Environmental Statistics, v.5, p. 240-256, 2000. 